\title{
Impacto del uso racional de antimicrobianos en una clínica de tercer nivel en Colombia
}

\author{
Christian J. Pallares y Juan C. Cataño
}

\section{Impact of rational use of antibiotics in a third level clinic in Colombia}

Background: The increasing resistance of bacteria to antibiotics threatens the survival of patients and health costs. Aim: To determine the impact of an antimicrobial stewardship program in bacterial resistance and antibiotic consumption. Materials and Methods: Quasi experimental study in a third level clinic in the city of Medellin, that evaluate in two time periods (pre-intervention between October 2012 and September 2013 and post intervention between October 2013 and September 2014) the impact of an antimicrobial stewardship program in terms of antibiotic consumption and bacterial ecology. Results: Adherence to institutional guidelines for management of infections in the post-intervention period was $82 \%$. Antibiotic consumption of meropenem, ceftriaxone, vancomycin and colistin decreased, and imipenem, daptomycin and linezolid was increased. A significant decrease in antibiotic resistance in Pseudomonas aeruginosa was observed, including carbapenems. An increase of extended spectrum beta lactamase production (ESBL) in Enterobacteriaceae (especially E. coli) and piperacillin/tazobactam resistance was observed. Conclusions: The construction and implementation of a strategy in hospitals with guidelines for managing infectious diseases, restrictions in antibiotic use, a permanent monitoring system for the formulation of antibiotics, achieved a positive impact on reducing antibiotic use and bacterial resistance.

Key words: Antibiotics, infectious diseases, epidemiological surveillance, hospital infection, bacterial resistance, defined daily dose.

Palabras clave: Antibióticos, enfermedades infecciosas, vigilancia epidemiológica, infección asociada a la atención en salud, resistencia bacteriana, dosis diaria definida.

\section{Introducción}

$\mathrm{E}$ 1 incremento en la resistencia de los microorganismos a los cada vez más escasos antimicrobianos que se tienen disponibles es un patrón generalizado en todo el mundo, lo que trae como consecuencia directa el aumento de la morbimortalidad y los costos a los sistemas de salud ${ }^{1,2}$.

Este es un problema médico y económico, ya que la infección por bacterias resistentes a múltiples antimicrobianos no sólo genera uso de moléculas más costosas y de mayor espectro antimicrobiano, sino que aumenta el riesgo de falla terapéutica y muerte, prolongando además la estancia hospitalaria, lo que encarece la atención en salud ${ }^{3,4}$. Algunos patrones de resistencia bacteriana se han asociado al uso indiscriminado de antimicrobianos ${ }^{5,6}$, lo que hace necesaria la implementación de estrategias que promuevan su uso racional, con el fin de disminuir la presión selectiva no sólo sobre los patógenos intrahospitalarios, sino también sobre los que vienen de la comunidad; esto con el fin de disminuir el daño colateral de la multi-resistencia que, a su vez, reducirá los costos de la atención en salud ${ }^{7,8}$.

El uso apropiado de antimicrobianos está fundamentado en la utilización del antimicrobiano más efectivo, con el menor número de efectos adversos, por la vía de administración más fácil y al menor costo posible ${ }^{9,10}$. Bajo esta premisa, los infectólogos son los profesionales capacitados para poder dirigir toda una serie de medidas encaminadas a racionalizar el uso de las limitadas opciones de antimicrobianos de las que se dispone actualmente.

En países desarrollados se han realizado estudios que demuestran cómo un programa que promueva el uso racional de antimicrobianos genera un efecto positivo en los costos de la atención en salud, así como también en los patrones de susceptibilidad de los microorganismos aislados $^{11,12}$. En Colombia existe alguna evidencia que los programas de uso racional de antimicrobianos, con la implementación de estrategias de vigilancia y control están asociados a una disminución en el consumo de algunos de ellos, cambio en los patrones de resistencia
Centro Internacional de Entrenamiento e Investigaciones MédicasCIDEIM, Cali, Colombia (CJPG) Universidad de Antioquia y Clínica CES (Corporación para Estudios en Salud), Medellín, Colombia (JCCC).

Conflictos de interés: Ninguno que declarar.

Fuente de financiamiento: Ninguna.

Recibido: 16 de febrero de 2016 Aceptado: 23 de abril de 2017

Correspondencia a: Juan C. Cataño Correa kataju@hotmail.com 
bacteriana específicos a corto plazo (enterobacterias productoras de $\beta$-lactamasas de espectro extendido -BLEE- y Pseudomonas aeruginosa resistente a cefalosporinas de $4^{\text {a }}$ generación y quinolonas) y ahorro en los costos de los medicamentos ${ }^{13-15}$, además de una disminución significativa en el riesgo de muerte, como factor independiente en pacientes con infecciones asociadas a la atención en salud (IAAS) ${ }^{16}$.

El presente trabajo pretende evaluar el impacto que tiene un programa diseñado para racionalizar el uso de antimicrobianos, en términos de resistencia bacteriana y consumo de antimicrobianos en una clínica de tercer nivel de la ciudad de Medellín.

\section{Materiales y Métodos}

Estudio cuasi experimental, de temporalidad prospectiva, que comparó dos períodos de tiempo (pre intervención entre octubre de 2012 y septiembre de 2013 y post intervención entre octubre de 2013 y septiembre de 2014) en Clínica CES, una clínica de tercer nivel de la ciudad de Medellín. La institución hospitalaria en la cual se realizó el estudio contaba, en ese entonces, con 153 camas de hospitalización, 13 camas de cuidados especiales y 6 camas de cuidado intensivo (UCI) adultos.

Durante el primer año del estudio (período pre intervención) se recolectó información sobre el uso de antimicrobianos, ecología bacteriana e IAAS. El consumo de antimicrobianos se determinó usando el sistema de dosis diaria definida (DDD) utilizando el programa $\mathrm{ABC}$ calc -antibiotic consumption calculator- (Microsoft Excel ${ }^{\circledR}$ application versión Office 2010), construido producto de la división del consumo de un determinado antimicrobianos en gramos sobre la multiplicación de número de camas de la institución por la proporción de ocupación por el número de días del mes por la constante DDD (disponible en la página de la Organización Mundial de la Salud para cada antimicrobiano) por 100 camas día. La susceptibilidad de los microorganismos aislados de muestras clínicas se determinó utilizando el equipo automatizado Vitek-2® de BioMerieux, siguiendo los puntos de corte de CLSI 2014 (Clinical and Laboratory Standars Institute) ${ }^{18}$. Los casos de IAAS fueron determinados usando los criterios del CDC (Centers for Disease Control and Prevention) $)^{19}$ $\mathrm{y}$ mediante vigilancia activa prospectiva diaria.

La intervención fue definida como la aplicación de medidas dirigidas a racionalizar el uso de antimicrobianos siguiendo la guía internacional de implementación de programas de gerenciamiento de antimicrobianos ${ }^{17}$ y bajo la dirección de un médico infectólogo, quien, con una dedicación laboral de 4 h diarias, se encargó de implementar las siguientes políticas:
- Diseño de guías institucionales para el manejo de las principales patologías infecciosas de la clínica (infección del tracto urinario, neumonía, sepsis e infección de piel y tejidos blandos), buscando optimizar el diagnóstico y enfocando el manejo empírico con base a la epidemiología microbiológica local.

- Política de restricción de antimicrobianos, consistente en el despacho automático de la primera dosis formulada de carbapenémicos, vancomicina, fluconazol, tigeciclina, daptomicina y linezolid, requeriendo las dosis siguientes de la autorización de infectología; así se buscó desestimular el uso masivo de éstos y ejercer un control más cercano con respecto a la formulación de ellos.

- Análisis de los antibiogramas de los microorganismos aislados en la clínica, para definir junto al médico tratante la pertinencia del antimicrobiano y la mejor opción de tratamiento a utilizar según el caso en particular.

La recolección de los datos fue realizada por los departamentos de química farmacéutica, epidemiología hospitalaria y microbiología clínica. Para el análisis estadístico se realizó un análisis exploratorio de datos para conocer la tendencia de cada variable del estudio y para la identificación de datos erróneos o atípicos. El análisis descriptivo se realizó estableciendo proporciones para las variables cualitativas, media o medianas y su respectiva desviación estándar o rangos intercuartílicos, según la distribución de las variables cuantitativas. El proceso de descripción de variables categóricas se complementó con tablas de frecuencia con sus respectivos porcentajes. Para las variables cuantitativas se verificó la normalidad de los datos a través del test de Shapiro Wilk. Se aplicó la prueba de $\chi^{2}$, o la prueba exacta de Fisher (cuando los valores esperados en una o más casillas eran inferiores a cinco) para las variables de tipo cualitativo. Para las variables cuantitativas se usaron pruebas paramétricas o no paramétricas dependiendo de la normalidad en la distribución ( T de Student y Mann Whitney para comparación de dos grupos independientes, respectivamente), con el objetivo de evaluar diferencias estadísticamente significativas de las variables. Valores de $\mathrm{p}<0,05$ fueron considerados estadísticamente significativos. El procesamiento de los datos se realizó en el software estadístico STATA $®$ versión 10 .

De acuerdo a la normativa vigente en Colombia (resolución 8.430 de 1993, artículo 11), esta investigación fue considerada de riesgo mínimo debido a que se emplearon técnicas y métodos de investigación documental, y no se realizó intervención alguna o modificación intencionada de las variables biológicas, fisiológicas, sicológicas o sociales de los individuos que participaron en el estudio. 


\section{Resultados}

La adherencia a las guías institucionales para el manejo de la patología infecciosa en el período post-intervención fue de $82 \%$. Se observó un incremento no significativo en el consumo en DDD en el período post-intervención respecto al pre-intervención de $23 \%$ (p: 0,78), con una disminución en el consumo de meropenem, ceftriaxona, colistín y vancomicina (Figura 1a) y un incremento de daptomicina, tigeciclina, imipenem, aztreonam y linezolid (Figura 1b) en el período post intervención (Tabla 1, Figura 2). Respecto a la ecología bacteriana, se observó una disminución significativa en la resistencia de $P$. aeruginosa a antimicrobianos, incluyendo los carbapenémicos. Para enterobacteriaceas se observó un incremento en la resistencia a ceftriaxona, ampicilina/sulbactam y piperacilina/tazobactam (Tabla 2).

El promedio de la tasa de IAAS en el período postintervención fue significativamente menor comparado con el período pre-intervención $(3,6$ versus 7,7 p < 0,0001) (Figura 3). Respecto a la tasa de IAAS por dispositivos invasores, se observó una reducción significativa en infección del tracto urinario asociada a catéter vesical (ITU-AS) $(5,1$ versus $2,7 \mathrm{p}: 0,02)$, neumonía asociada a ventilación mecánica (NAVM) $(6,4$ versus 1,5 p: 0,001) y una reducción no significativa para infección del torrente sanguíneo asociado a catéter (ITS-AC) (4,0 versus 1,4 p: 0,33) (Figura 4).

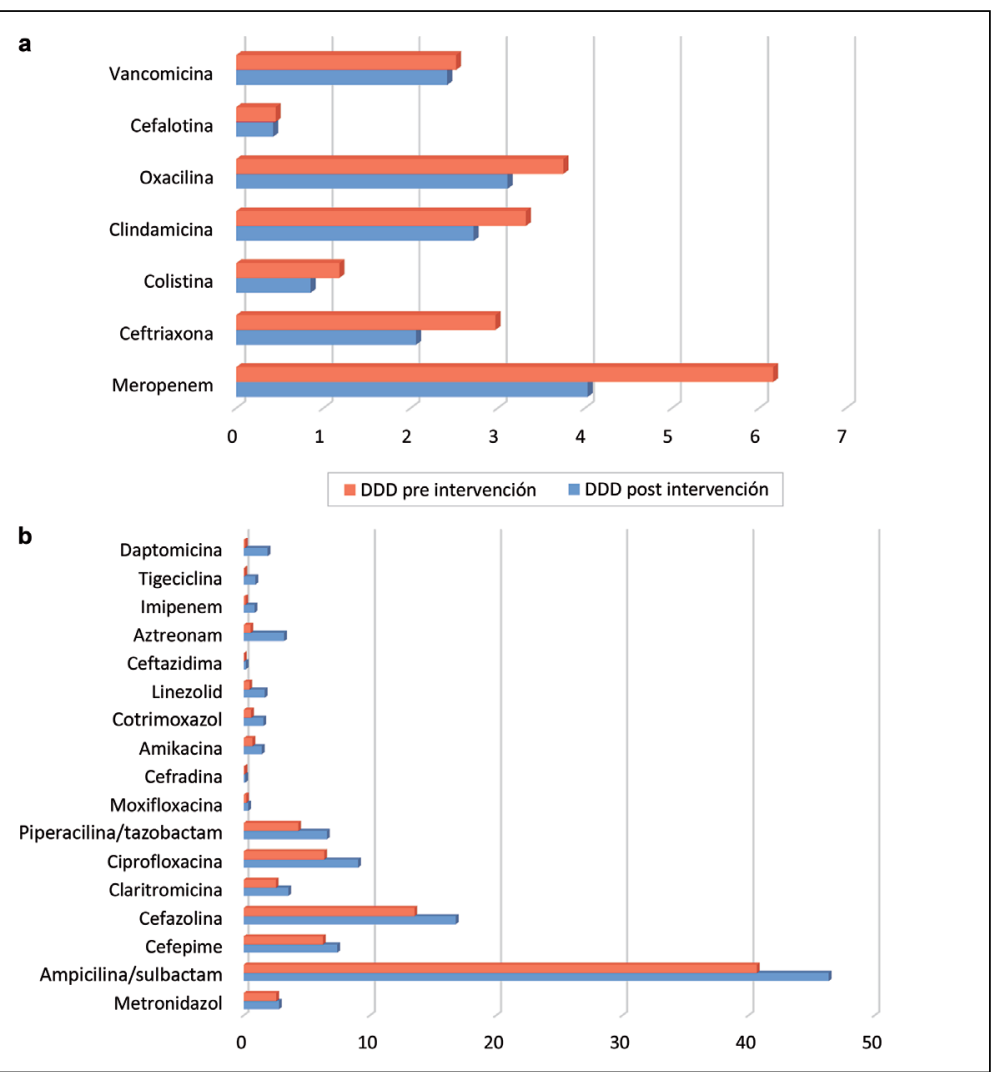

Figura 1. Dosis diaria definida (DDD x 100 camas día) para antimicrobianos durante el período pre- y post-intervención.

\begin{tabular}{|c|c|c|c|c|}
\hline Antimicrobiano & DDD pre-intervención & DDD post-intervención & Cambio en el consumo & $p^{*}$ \\
\hline Ampicilina/sulbactam & 40,67 & 46,36 & $+14 \%$ & 0,12 \\
\hline Oxacilina & 3,75 & 3,11 & $17 \%$ & 0,17 \\
\hline Piperacilina/tazobactam & 4,31 & 6,59 & $+53 \%$ & $>0,001$ \\
\hline Cefazolina & 13,52 & 16,79 & $+24 \%$ & 0,003 \\
\hline Ceftriaxona & 2,97 & 2,06 & $-31 \%$ & 0,02 \\
\hline Cefepime & 6,27 & 7,41 & $+18 \%$ & 0,23 \\
\hline Imipenem & 0,14 & 0,85 & $+507 \%$ & 0,001 \\
\hline Meropenem & 6,16 & 4,03 & $-35 \%$ & $>0,001$ \\
\hline Aztreonam & 0,54 & 3,2 & $+493 \%$ & $>0,001$ \\
\hline Amikacina & 0,69 & 1,42 & $+106 \%$ & $>0,001$ \\
\hline Ciprofloxacina & 6,37 & 9,06 & $+42 \%$ & 0,009 \\
\hline Vancomicina & 2,52 & 2,42 & $-4 \%$ & 0,88 \\
\hline Daptomicina & 0,1 & 1,89 & $+1790 \%$ & $>0,001$ \\
\hline Tigeciclina & 0,05 & 0,93 & $+1760 \%$ & $>0,001$ \\
\hline Clindamicina & 3,32 & 2,72 & $-18 \%$ & 0,13 \\
\hline Linezolid & 0,44 & 1,66 & $+277 \%$ & $>0,001$ \\
\hline Colistín & 1,18 & 0,85 & $-28 \%$ & 0,38 \\
\hline
\end{tabular}




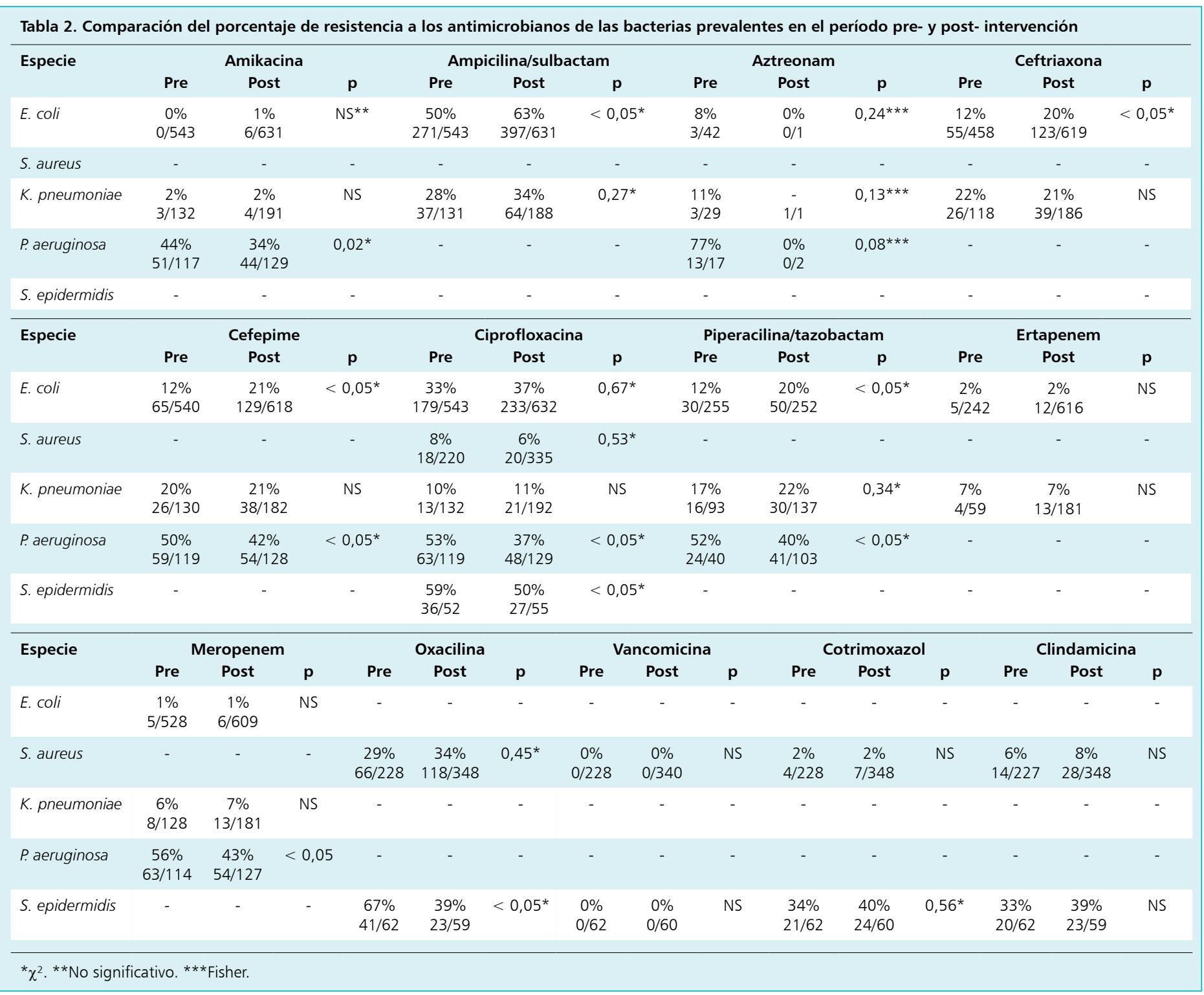

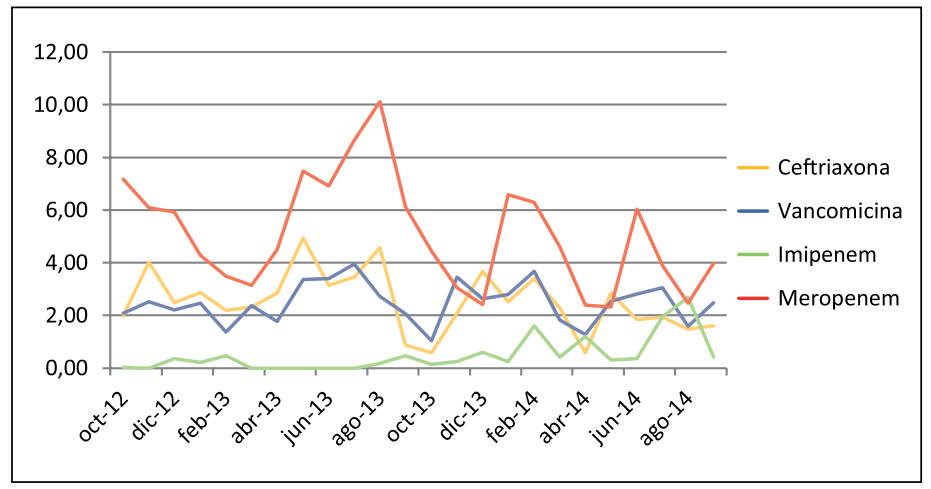

Figura 2. Dosis diaria definida (DDD x 100 camas día) para antimicrobianos controlados desde octubre de 2012 hasta septiembre de 2014.

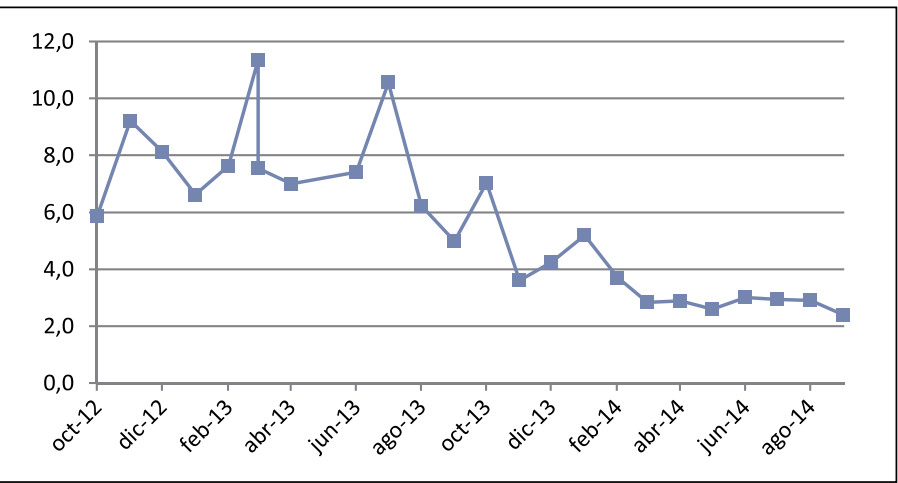

Figura 3. Tasa de infección asociada a la atención en salud (IAAS) x 1.000 días paciente, desde octubre de 2012 hasta septiembre de 2014. 


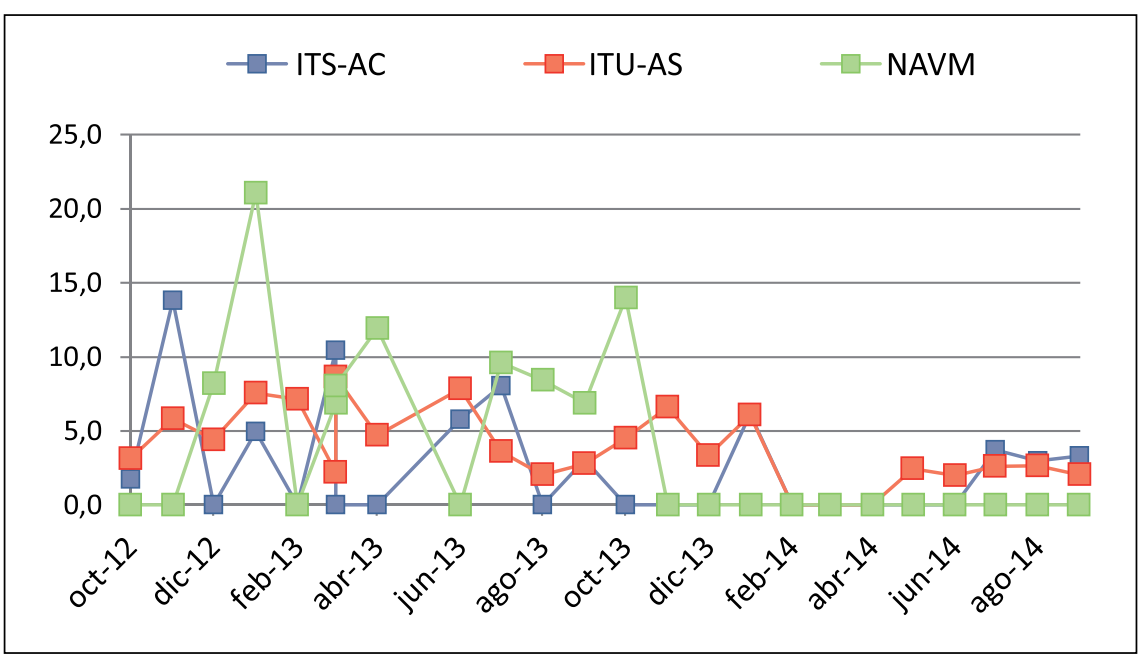

Figura 4. Tasa de IAAS por dispositivos invasores (x 1.000 días dispositivo) desde octubre de 2012 hasta septiembre de 2014. ITS-AC: infección del torrente sanguíneo asociada a catéter. ITU-AS: infección del tracto urinario asociada a sonda. NAVM: neumonía asociada a ventilación mecánica.

\section{Discusión}

Una de las formas más eficientes para enfrentar la resistencia a antimicrobianos en los microorganismos hospitalarios es la implementación de programas que promuevan su uso racional mediante la implementación de guías de tratamiento para las patologías infecciosas más frecuentes $s^{5,710,12,17}$. Esto, acompañado del seguimiento al consumo de antimicrobianos en forma controlada, incluyendo restricción de ciertos antimicrobianos de acuerdo a la ecología hospitalaria y sugerencias de cambio en el tratamiento dirigidas por un experto, garantiza un impacto en el consumo, ya que son los factores modificables más importantes en la práctica clínica con respecto al uso de antimicrobianos en una institución. La adherencia a la guía institucional para el manejo de la patología infecciosa fue superior a $80 \%$ y estuvo asociada a la disminución en el consumo de antimicrobianos frecuentemente usados en el ambiente hospitalario como meropenem. Este hallazgo puede estar directamente relacionado con la disminución en la resistencia a antimicrobianos, en general, para $P$. aeruginosa, bacteria que por presión selectiva secundaria al uso indiscriminado de este tipo de antimicrobianos adquiere resistencia ${ }^{23}$.

Es importante mencionar que la disminución en el consumo de meropenem está relacionada, en cierta forma, con el incremento del consumo de imipenem; esto, atribuible a la creciente resistencia específica a meropenem que se venía observando en la clínica, razón por la que se decidió entonces restringir este antimicrobiano dando la opción de utilizar imipenem como alternativa en carbapenémicos. Aunque la tendencia en el consumo antimicrobiano fue hacia un aumento significativo en su prescripción, esta situación se explica porque durante el transcurso del estudio, la institución se convirtió en un centro de referencia en enfermedades infecciosas, aumentando la complejidad de los pacientes admitidos; ello repercutió necesariamente en su vulnerabilidad frente a las infecciones y necesidad de usar antimicrobianos.

Ceftriaxona, una de las moléculas más controladas durante la intervención debido a su capacidad de seleccionar microbiota bacteriana resistente -especialmente aquella productora de $\mathrm{BLEE}^{24,25}$ - mostró una disminución significativa en el consumo. A pesar de esto, se observó que en el período post-intervención se incrementó la proporción de enterobacteriaceas productoras de BLEE (principalmente Escherichia coli) y resistencia a piperacilina/tazobactam. Este hallazgo puede estar relacionado con el aumento significativo en el consumo de aztreonam y piperacilina/tazobactam en el período post-intervención; la evidencia ha demostrado que el incremento en el uso de estos antimicrobianos selecciona para este tipo de microorganismos resistentes ${ }^{24,26}$.

La disminución en el consumo de vancomicina estuvo relacionada con que el producto innovador de este antimicrobiano no se encuentra disponible en Colombia y los genéricos de vancomicina han mostrado en nuestro país problemas de fármaco-equivalencia en términos de eficacia clínica, así como reporte de fallas terapéuticas ${ }^{27,28}$; vancomicina sólo se avaló para profilaxis pre-quirúrgica, razón por la cual se observó un incremento en el consumo de linezolid y daptomicina como opciones alternativas.

Durante la implementación del programa de uso racional de antimicrobianos se observó una disminución en la incidencia de las IAAS. Aunque esto puede estar relacionado con el programa de uso racional de antimicrobianos, este hallazgo no puede ser definido como un resultado del programa, sino como resultado de estrategias propias del control de las IAAS realizadas en este mismo período de tiempo (como la inclusión en la política de una 
constante vigilancia en la formulación de las profilaxis quirúrgicas que evitó dar tratamiento antimicrobiano a colonizaciones, la vigilancia de la pertinencia del uso de dispositivos invasores y las buenas prácticas en la atención por parte del personal de la salud).

Dentro de las limitaciones del presente estudio, es importante mencionar que no se tienen datos acerca de las co-morbilidades de los pacientes atendidos durante ambos períodos, lo cual podría modificar en cierta forma el efecto de la intervención, especialmente desde la probabilidad del riesgo de adquirir una IAAS. Tampoco fue posible realizar un análisis de costos, debido a la regulación por el sistema de salud durante el período del estudio de los precios y la inclusión de copias y genéricos de un mismo principio activo.

\section{Conclusión}

Teniendo en cuenta los hallazgos del estudio, es importante considerar en las instituciones hospitalarias la construcción e implementación de una estrategia para combatir el uso indiscriminado de antimicrobianos y la resistencia bacteriana constituida por guías de manejo de la patología infecciosa basadas en los patrones microbiológicos de cada entidad de salud, restricción en el uso de algunos antimicrobianos y un sistema de vigilancia y monitoria permanente a la formulación; esto asociado a estrategias en prevención y control de infecciones puede lograr un cambio conductual positivo en el personal de salud.

Agradecimientos. Los autores queremos agradecer a Marcela Gaviria (Microbióloga de la Clínica CES), Isabel Macías (Jefe de Vigilancia epidemiológica Clínica CES) y Alejandra Rendón (Química Farmacéutica Clínica CES) por su invaluable ayuda en la realización del presente estudio y de nuestra labor diaria.

\section{Resumen}

Introducción: El aumento progresivo de la resistencia bacteriana a antimicrobianos amenaza la sobrevida de los pacientes y los costos en salud. Objetivo: Determinar el impacto de un programa para el uso racional de antimicrobianos en resistencia bacteriana y consumo de antimicrobianos en una institución de tercer nivel de atención. Materiales y Métodos: Estudio cuasi experimental realizado en una clínica de tercer nivel de la ciudad de Medellín, donde se comparó en dos períodos de tiempo (pre intervención entre octubre de 2012 y septiembre de 2013 y post intervención entre octubre de 2013 y septiembre de 2014) el consumo y la resistencia a antimicrobianos de las bacterias hospitalarias. Resultados: La adherencia a las guías institucionales para el manejo de la patología infecciosa en el período post intervención fue $82 \%$. Se observó una disminución en el consumo de meropenem, ceftriaxona, colistín, vancomicina, y un incremento en el consumo de imipenem, daptomicina y linezolid. Se observó una disminución significativa en la resistencia de Pseudomonas aeruginosa a los antimicrobianos, incluyendo los carbapenémicos. Se presentó un incremento en la proporción de enterobacteriaceas productoras de BLEE (principalmente Escherichia coli) y resistencia a piperacilina/tazobactam. Conclusiones: La construcción e implementación en las instituciones hospitalarias de una estrategia conformada por guías de manejo de la patología infecciosa, restricción en el uso de antimicrobianos y un sistema de vigilancia y un monitoreo permanente a la formulación de éstos, logra un impacto positivo en disminución del consumo de antimicrobianos y resistencia bacteriana.

\section{Referencias bibliográficas}

1.- Michael C A, Dominey-Howes D, Labbate $\mathrm{M}$. The antimicrobial resistance crisis: causes, consequences, and management. Front Public Health 2014; 2: 145.

2.- Choudhury R, Panda S, Singh D V. Emergence and dissemination of antibiotic resistance: a global problem. Indian J Med Microbiol 2012; 30 (4): 384-90.

3.- Cantón R, Horcajada J P, Oliver A, Garbajosa $P R$, Vila J. Inappropriate use of antibiotics in hospitals: the complex relationship between antibiotic use and antimicrobial resistance. Enferm Infecc Microbiol Clin 2013; 31 Suppl 4: 3-11.

4.- Sipahi O R. Economics of antibiotic resistance.
Expert Rev Anti Infect Ther 2008; 6 (4): 52339.

5.- Goff D A. Antimicrobial stewardship: bridging the gap between quality care and cost. Curr Opin Infect Dis 2011; Suppl 1: S11-20.

6.- Kollef M H, Fraser V J. Antibiotic resistance in the intensive care unit. Ann Intern Med 2001; 134 (4): 298-314.

7.- Davey P, Brown E, Charani E, Fenelon L, Gould I M, Holmes A, et al. Interventions to improve antibiotic prescribing practices for hospital inpatients. Cochrane Database Syst Rev 2013; 4: CD003543.

8.- Smith T, Philmon C L, Johnson G D, Ward W S, Rivers L L, Williamson S A, et al. Antimicrobial stewardship in a community hospital: attacking the more difficult problems.
Hosp Pharm 2014; 49: 839-46.

9.- Llor C, Bjerrum L. Antimicrobial resistance: risk associated with antibiotic overuse and initiatives to reduce the problem. Ther Adv Drug Saf 2014; 5: 229-41.

10.- Nilholm H, Holmstrand L, Ahl J, Månsson F, Odenholt I, Tham J, et al. An auditbased, infectious disease specialist-guided antimicrobial stewardship program profoundly reduced antibiotic use without negatively affecting patient outcomes. Open Forum Infect Dis 2015; 2 (2): ofv042.

11.- Avent M L, Hall L, Davis L, Allen M, Roberts J A, Unwin S, et al. Antimicrobial stewardship activities: a survey of Queensland hospitals. Aust Health Rev 2014; 38: 557-63.

12.- Trivedi K K, Dumartin C, Gilchrist M, 
Wade P, Howard P. Identifying best practices across three countries: hospital antimicrobial stewardship in the United Kingdom, France, and the United States. Clin Infect Dis 2014; 59 Suppl 3: S170-8.

13.- Cataño J. The economic and ecologic impact of infectious diseases specialists in hospitals. Acta Med Col 2008; 33: 58-62.

14.- Cataño J, Castaño O. Evaluation of the impact of a program of epidemiological surveillance in consumption of antibiotics and flora in a third level clinic. Infectio 2009; 13: 6-13.

15.- Pallares C J, Martínez E. Implementation of a regulated antibiotic use program in two medical-surgical intensive units care in a third level mayor teaching hospital in Colombia. Infectio 2012; 16 (4): 192-8.

16.- Pallares C J, Martínez E. Mortality risk factors associated with healthcare infections in a tertiary level university hospital in Colombia. Biomédica 2014; 34 (Suppl 1): 148-55.

17.- Barlam T F, Cosgrove S E, Abbo L M, MacDougall C, Schuetz A N, Septimus E J, et al. Implementing an antibiotic stewardship program: Guidelines by the Infectious Diseases Society of America and the Society for Healthcare Epidemiology of America. Clin Infect Dis. 2016 May 15; 62 (10): e51-77.

18.- CLSI. Performance standards for antimicrobial susceptibility testing; Twenty-fourth informational supplement. CLSI document M100-S24. Wayne, PA: Clinical and Laboratory Standards Institute; 2014.

19.- Horan T C, Andrus M, Dudeck M A. CDC/ NHSN surveillance definition of health careassociated infection and criteria for specific types of infections in the acute care setting. Am J Infect Control 2008; 36: 309-32.

20.- Huttner B, Harbarth S, Nathwani D. ESCMID Study Group for Antibiotic Policies (ESGAP). Success stories of implementation of antimicrobial stewardship: a narrative review. Clin Microbiol Infect 2014; 20: 954-62.

21.- Pulcini C, Mainardi J L. Antimicrobial stewardship: an international emergency. Clin Microbiol Infect 2014; 20: 947-8.

22.- Wagner B, Filice G A, Drekonja D, Greer N, MacDonald R, Rutks I, et al. Antimicrobial stewardship programs in inpatient hospital settings: a systematic review. Infect Control Hosp Epidemiol 2014; 35: 1209-28.

23.- Pena C, Guzmán A, Suárez C, Domínguez M A, Tubau F, Pujol M, et al. Effects of carbapenem exposure on the risk for digestive tract carriage of intensive care unit-endemic carbapenemresistant Pseudomonas aeruginosa strains in critically ill patients. Antimicrob Agents Chemother 2007; 51 (6): 1967-71.

24.- Paterson D L, Ko W C, Von Gottberg A, Mohapatra S, Casellas J M, Goossens H, et al.
International prospective study of Klebsiella pneumoniae bacteremia: implications of extended-spectrum, beta-lactamase production in nosocomial infections. Ann Intern Med 2004; 140 (1): 26-32.

25.- Cassier $\mathrm{P}$, Lallechère $\mathrm{S}$, Aho $\mathrm{S}$, Astruc $\mathrm{K}$, Neuwirth C, Piroth L, et al. Cephalosporin and fluoroquinolone combinations are highly associated with CTX-M beta-lactamaseproducing E. coli: a case-control study in a French teaching hospital. Clin Microbiol Infect 2011; 17(11): 1746-51.

26.- Dinubile M J, Friedland I, Chan C Y, Moltyl M R, Giezek H, Shiveprakash M, et al. Bowel colonization with resistant gram negative bacilli after antimicrobial therapy of intra-abdominal infections: observations from two randomized comparative clinical trials of ertapenem therapy. Eur J Clin Microbiol Infect Dis 2005; 24 (7): 443-9.

27.- Vesga $\mathrm{O}$, Agudelo M, Salazar B, Rodríguez C A, Zuluaga A F. Generic vancomycin products fail in vivo despite being pharmaceutical equivalents of the innovator. Antimicrob Agents Chemother 2010; 54: 3271-79.

28.- Rodríguez C A, Agudelo M, Cataño J C, Zuluaga A, Vesga O. Potential therapeutic failure of generic vancomycin in a liver transplant patient with MRSA peritonitis and bacteremia. J Infect 2009; 59: 277-80. 